\title{
THE FIRST ICRANET CATALOG OF BINARY-DRIVEN HYPERNOVAE
}

\author{
G. B. Pisani ${ }^{1,2}$, R. Ruffini ${ }^{1,2,3,4}$, Y. Aimuratov ${ }^{1,2,3}$, C. L. Bianco ${ }^{1,2}$, M. Karlica $^{1,2,3}$, M. KovaceviC ${ }^{1,2,3}$, R. Moradi $^{1,2}$,

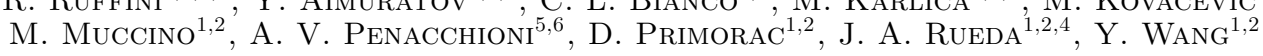 \\ Draft version September 18, 2017
}

\begin{abstract}
In a series of recent publications, scientists from ICRANet, led by professor Remo Ruffini, have reached a novel comprehensive picture of gamma-ray bursts (GRBs) thanks to their development of a series of new theoretical approaches. Among those, the induced gravitational collapse (IGC) paradigm explains a class of energetic, long-duration GRBs associated with Ib/c supernovae (SN), recently named binary-driven hypernovae (BdHNe).

BdHNe have a well defined set of observational features which allow to identify them. Among them, the main two are: 1) long duration of the GRB explosion, namely larger than $2 \mathrm{~s}$ in the rest frame; 2) a total energy, released in all directions by the GRB explosion, larger than $10^{52}$ ergs.

A striking result is the observation, in the BdHNe sources, of a universal late time power-law decay in the X-rays luminosity after $10^{4} \mathrm{~s}$, with typical decaying slope of $\sim 1.5$. This leads to the possible establishment of a new distance indicator having redshift up to $z \sim 8$.

Thanks to this novel theoretical and observational understanding, it was possible for ICRANet scientists to build the firstst BdHNe catalog, composed by the 345 BdHNe identified up to the end of 2016.

Keywords: supernovae: general — binaries: general — gamma-ray burst: general — stars: neutron
\end{abstract}

\section{TOWARDS A FIRST CATALOG OF BINARY-DRIVEN HYPERNOVAE}

The first observations by the BATSE instrument on board the Compton $\gamma$-ray Observatory satellite have evidenced what has later become known as the prompt radiation of GRBs. On the basis of their hardness as well as their duration, GRBs were initially classified into short and long in an epoch when their cosmological nature was still being debated (Mazets et al. 1981; Klebesadel 1992; Dezalay et al. 1992; Kouveliotou et al. 1993; Tavani 1998).

The advent of the BeppoSAX satellite (Boella et al. 1997) introduced a new approach to GRBs by introducing joint observations in the X-rays and $\gamma$-rays thanks to its instruments: the Gamma-ray Burst Monitor (40$700 \mathrm{keV})$, the Wide Field Cameras (2-26 keV), and the Narrow Field Instruments (2-10 keV). The unexpected discovery of a well separate component in the GRB soon appeared: the afterglow, namely a radiation lasting up to $10^{5}-10^{6} \mathrm{~s}$ after the emission of the prompt radiation (see Costa et al. 1997a,b; Frontera et al. 1998, 2000; de Pasquale et al. 2006). Beppo-SAX clearly indicated the existence of a power law behavior in the late X-ray emission (LXRE).

The coming of the Swift satellite (Gehrels et al. 2004; Evans et al. 2007, 2010), significantly extending the ob-

\footnotetext{
${ }^{1}$ Dipartimento di Fisica, Sapienza Università di Roma and ICRA, Piazzale Aldo Moro 5, I-00185 Roma, Italy

2 International Center for Relativistic Astrophysics Network (ICRANet), Piazza della Repubblica 10, I-65122 Pescara, Italy

${ }^{3}$ Université de Nice Sophia-Antipolis, Grand Château Parc Valrose, Nice, CEDEX 2, France

${ }^{4}$ ICRANet-Rio, Centro Brasileiro de Pesquisas Fisicas, Rua Dr. Xavier Sigaud 150, Rio de Janeiro, RJ, 22290-180, Brazil

5 University of Siena, Department of Physical Sciences, Earth and Environment, Via Roma 56, I-53100 Siena, Italy

6 ASI Science Data Center, via del Politecnico s.n.c., I-00133 Rome Italy
}

servation energy band to the X-ray band thanks to its X-ray Telescope (XRT band: $0.3-10 \mathrm{keV}$ ), has allowed us for the first time to uncover the unexplored region between the end of the prompt radiation and the power-law late X-ray behavior discovered by BeppoSAX: in some long GRBs, a steep decay phase was observed leading to a plateau followed then by a typical LXRE power law behavior (Evans et al. 2007, 2010).

Recently, Pisani et al. (2013) noticed the unexpected result that the LXREs of a "golden sample" (GS) of six long, closeby $(z \lesssim 1)$, energetic $\left(E_{\text {iso }}>10^{52} \mathrm{erg}\right) \mathrm{GRBs}$, when moved in the rest-frame of the sources, were showing a common power-law behavior (see Fig. 1), independently from the isotropic energy $E_{\text {iso }}$ coming from the GRB prompt radiation (see Fig 2). More unexpected was the fact that the plateau luminosity and duration before merging in the common LXRE power-law behavior were clearly functions of the $E_{\text {iso }}$ (see Fig. 2, and Ruffini et al. 2014c), while the late power-law remains independent from the $E_{\text {iso }}$ of the prompt emission (see Fig. 1-2, and Pisani et al. 2013; Ruffini et al. 2014c). For this reason, this striking scaling law has been used as a distance indicator to independently estimate the cosmological redshift of some long GRBs by imposing the overlap of their LXRE (see, e.g., Penacchioni et al. 2012, 2013; Ruffini et al. 2013b,c, 2014a), and also to predict, ten days in advance, the observation of the typical optical signature of the supernova SN 2013cq, associated with GRB 130427A (Ruffini et al. 2015, 2013a; de Ugarte Postigo et al. 2013; Levan et al. 2013).

All these analyses are based on the paradigms introduced in Ruffini et al. (2001a) for the space-time parametrization of the GRB phenomena, in Ruffini et al. (2001b) for the interpretation of the structure of the GRB prompt radiation, and in Ruffini et al. (2001c) for the induced gravitational collapse (IGC) mechanism, further 


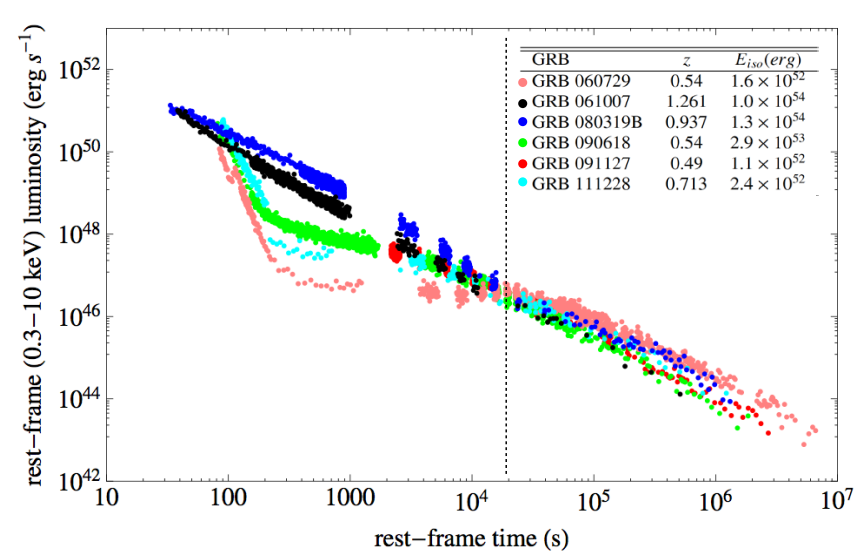

Figure 1. Scaling law found in the isotropic X-ray late times luminosity within the GS by Pisani et al. (2013). Despite the different early behavior, the different light curves join all together the same power law after a rest-frame time of $t_{r f} \sim 2 \times 10^{4} \mathrm{~s}$.

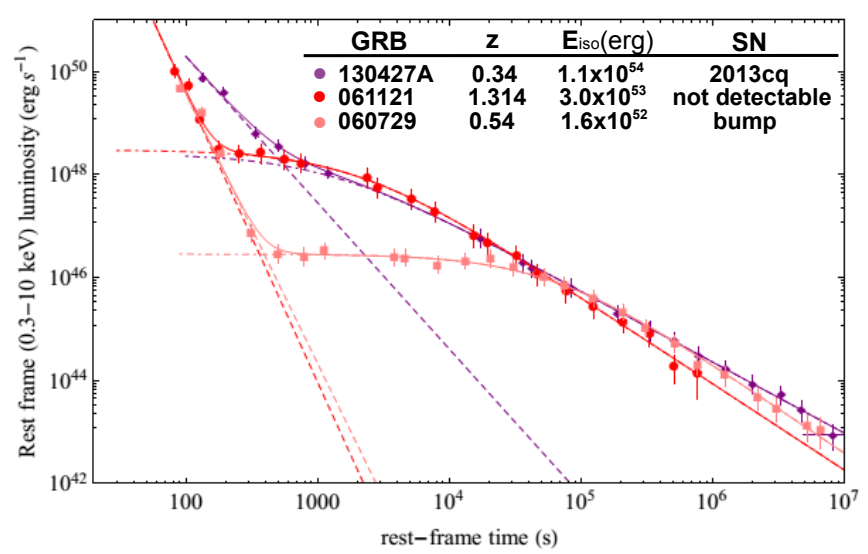

Figure 2. Nested structure of the isotropic X-ray luminosity of the BdHNe. This includes the previously mentioned scaling law of the late power law and leads to an inverse proportionality between the luminosity of the plateau and the rest-frame time delimiting its end and the beginning of the late power law decay Ruffini et al. (2014c).

developed in Ruffini et al. (2007), Rueda \& Ruffini (2012), Fryer et al. (2014), and Ruffini et al. (2016). In the present case, the phenomenon points to an IGC occurring when a tight binary system composed of a carbonoxygen core $\left(\mathrm{CO}_{\text {core }}\right)$ undergoes a supernova $(\mathrm{SN})$ explosion in the presence of a binary neutron star (NS) companion (Ruffini et al. 2001b, 2007; Izzo et al. 2012; Rueda \& Ruffini 2012; Fryer et al. 2014; Ruffini et al. 2015). When the IGC leads the NS to accrete enough matter and therefore to collapse to a black hole $(\mathrm{BH})$, the GRB shows a long duration, and its prompt emission overtakes the treshold value of $10^{52}$ ergs. The overall observed phenomenon is called binary-driven hypernova (BdHN; Fryer et al. 2014; Ruffini et al. 2015, 2016).

A decisive further step has been the identification as a BdHN of GRB 090423 (Ruffini et al. 2014b) at the extremely high redshift of $z=8.2$ (Salvaterra et al. 2009; Tanvir et al. 2009). On top of that, the LXRE of GRB 090423 overlaps entirely with the ones of the GS (see Fig. 3 ), extending such a scaling law up to extreme cosmological distances. This result led to the necessity of checking such an common behavior of the LXREs in BdHNe at redshifts larger than $z \sim 1$.

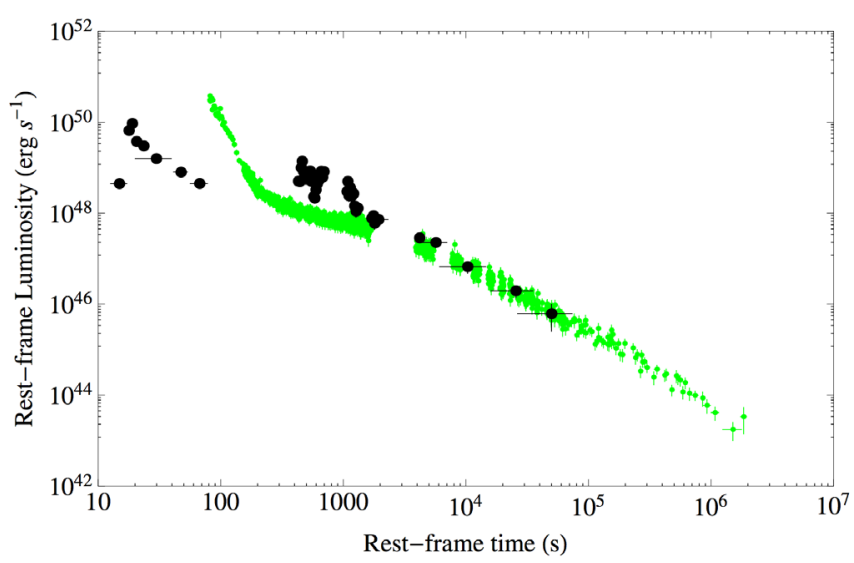

Figure 3. X-ray luminosity of GRB 090423 (black points) compared with the one of GRB 090618 (green points), the prototype BdHN, by Ruffini et al. (2014b).

In Pisani et al. (2016), we present an "enlarged sample" (ES) of 161 BdHNe observed up to the end of 2015. In this work we analysed the signatures contained in the LXREs at $t_{r f} \gtrsim 10^{4} \mathrm{~s}$, where $t_{r f}$ is the rest-frame time after the initial GRB explosion. In particular, we probed a further improvement for the presence of such an LXRE universal behavior of BdHNe by the introduction of a collimation effect within the emission mechanism.

In our recent work (Ruffini et al. 2017), we focused on analyzing the early X-Ray Flares in the GRB flareplateau-afterglow (FPA) phase observed by Swift/XRT. The FPA occurs only in the BdHNe while is not present in the other subclasses of GRBs, for details see Ruffini et al. (2016). The sample presented in Table 9 of Ruffini et al. (2017), namely an updated version of the ES up to the end of 2016, together with the BdHNe lacking LXRE data and the ones from the pre-Swift-era, counts $345 \mathrm{BdHNe}$ in total. This represents the current BdHNe catalog from ICRANet.

In the following, we present various insightful results which ICRANet scientists gained from this catalog: in Sections 2, 3, and 4, we describe how we built the ES and we study the LXRE features within it; finally, in Section 5 we refer to our up-to-date catalog of BdHNe and we draw our perspectives.

\section{THE FIRST ENLARGED SAMPLE OF BDHN}

Starting from the GS originally presented in Pisani et al. (2013), in Pisani et al. (2016) we have built a new sample of BdHNe, which we called "enlarged sample" (ES), under the following selection criteria:

- measured redshift $z$;

- GRB rest-frame duration larger than $2 \mathrm{~s}$;

- isotropic energy $E_{i s o}$ larger than $10^{52} \mathrm{erg}$; and

- presence of associated Swift/XRT data lasting at least up to $t_{r f}=10^{4} \mathrm{~s}$.

We collected 161 sources, satisfying our criteria, which cover 11 years of Swift/XRT observations, up to the end of 2015, see Table 2 of Pisani et al. (2016). The $E_{\text {iso }}$ of each source has been estimated using the observed redshift $z$ together with the best-fit parameters of the 


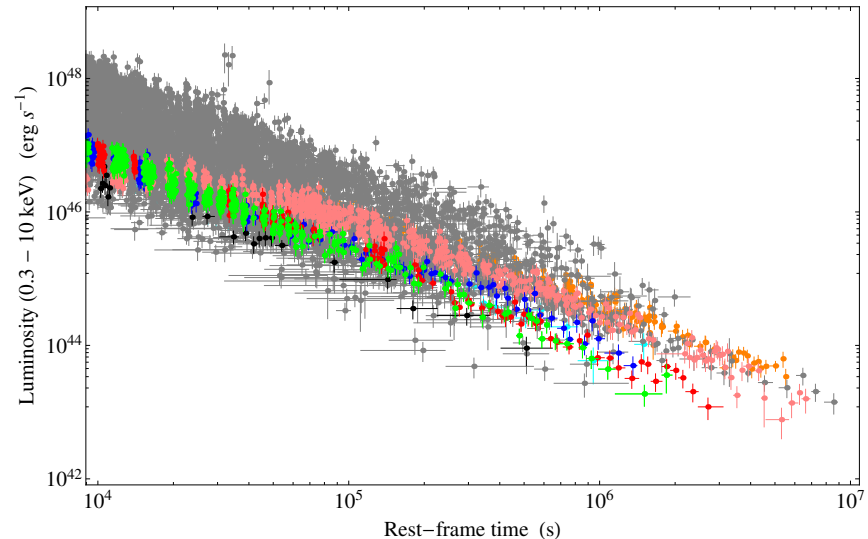

(a)

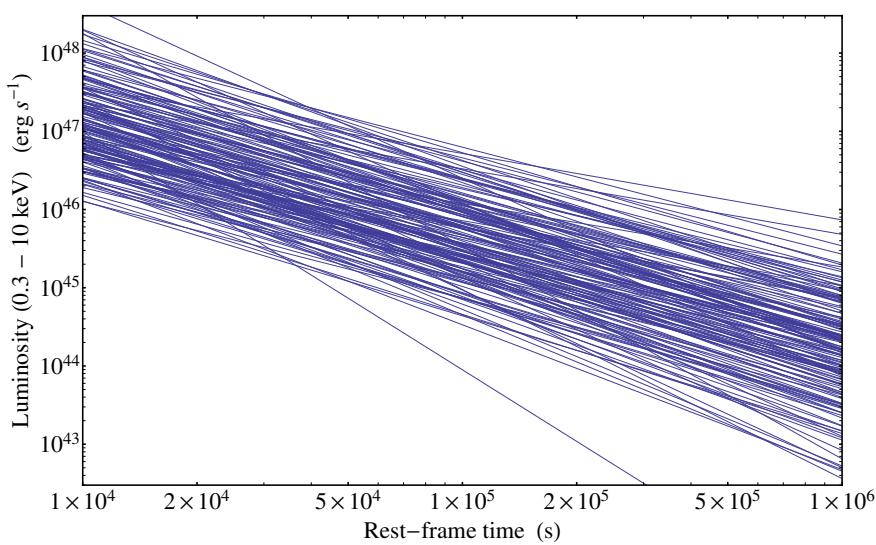

(b)

Figure 4. Panel (a): LXRE luminosity light curves of all 161 sources of the ES (gray) compared with the ones of the GS: GRB 060729 (pink), GRB 061007 (black), GRB 080913B (blue), GRB 090618 (green), GRB 091127 (red), and GRB 111228 (cyan), plus GRB 130427A (orange; from Pisani et al. 2016). Panel (b): power laws which best fit the luminosity light curves of the X-ray emissions of all 161 sources of the ES (from Pisani et al. 2016).

$\gamma$-ray spectrum published in the GCN circular archive ${ }^{7}$. Most of of the ES sources, 102 out of 161, have $\gamma$-ray data observed by Fermi/GBM and Konus-WIND, which, with their energy bands being 10-1000 keV and 20-2000 $\mathrm{keV}$, respectively, lead to a solid estimate of the $E_{i s o}$, computed in the "bolometric" $1-10^{4} \mathrm{keV}$ band (Bloom et al. 2001). The remaining sources of the ES have had their $\gamma$-ray emission provided by Swift/BAT only, with the unique exception of one source observed by HETE. The energy bands of these two detectors, being 15-150 $\mathrm{keV}$ and 8-400 keV, respectively, lead to an estimate of $E_{\text {iso }}$ by extrapolation in the "bolometric" $1-10^{4} \mathrm{keV}$ band (Bloom et al. 2001).

\section{GOING TO THE REST-FRAME}

We compare the Swift/XRT isotropic luminosity light curve $L_{i s o}\left(t_{r f}\right)$ for 161 GRBs of the ES in the common rest-frame energy range of $0.3-10 \mathrm{keV}$. We initially adjust the observed Swift/XRT flux $f_{\text {obs }}$ as if it had been observed in the $0.3-10 \mathrm{keV}$ rest-frame energy range. In the detector frame, the $0.3-10 \mathrm{keV}$ rest-frame energy band becomes $[0.3 /(1+z)]-[10 /(1+z)] \mathrm{keV}$, where $z$ is the measured redshift of the GRB. We assume a simple power-law as the best fit for the spectral energy distribution of the Swift/XRT data ${ }^{8}$ :

$$
\frac{d N}{d A d t d E} \propto E^{-\gamma}
$$

Hence, we can calculate the flux light curve in the $0.3-$ $10 \mathrm{keV}$ rest-frame energy band, $f_{r f}$, multiplying the observed one, $f_{\text {obs }}$, by the k-correctionr:

$$
f_{r f}=f_{o b s} \frac{\frac{\int_{\underline{0.3 \mathrm{keV}}}^{\frac{10 \mathrm{keV}}{1+z}}}{1+\mathrm{keV}} E^{1-\gamma} d E}{\int_{0.3 \mathrm{keV}}^{10 \mathrm{keV}} E^{1-\gamma} d E}=f_{o b s}(1+z)^{\gamma-2} .
$$

Then, to calculate the isotropic X-ray luminosity $L_{i s o}$, we need to multiply $f_{r f}$ by the spherical surface having the luminosity distance as radius

$$
L_{i s o}=4 \pi d_{l}^{2}(z) f_{r f}
$$

7 http://gcn.gsfc.nasa.gov/gcn3_archive.html

8 http://www.swift.ac.uk/ where we assume a standard cosmological $\Lambda$ CDM model, namely $\Omega_{m}=0.27$ and $\Omega_{\Lambda}=0.73$. In the end, we convert the observed times into rest-frame times $t_{r f}$ :

$$
t_{r f}=\frac{t_{o b s}}{1+z} .
$$

After, we fit the isotropic luminosity light-curve late phase with a decaying power-law function defined as:

$$
L_{i s o}\left(t_{r f}\right)=L_{0} t_{r f}^{-\alpha}
$$

where $\alpha$, the power law index, is a positive number, and $L_{0}$ is the luminosity at a fixed time $t_{r f}=t_{0}$ after the GRB initial explosion in the rest-frame of the source. All the power-laws are shown in Fig. 4b. Fig. 5a shows the distribution of the $\alpha$ indexes within the ES. This distribution follows a Gaussian behavior having a mean value of $\mu_{\alpha}=1.48$ and a standard deviation of $\sigma_{\alpha}=0.32$. The LXRE luminosity light curves of the ES in the 0.3 $10 \mathrm{keV}$ rest-frame energy range are plotted in Fig. 4a, together with the curves of the GS. Fig. 4a shows that the power-laws within the ES span around two orders of magnitude in luminosity. The spread of the LXRE light curves in the ES is better shown off by Fig. 5b which display the distribution within the ES of the LXRE integrated energies $E_{L T}$ defined as:

$$
E_{L T} \equiv \int_{10^{4} s}^{10^{6} s} L_{i s o}\left(t_{r f}\right) \mathrm{d} t_{r f} .
$$

The solid red line in Fig. 5b is the Gaussian function that best fits the late integrated energies $E_{L T}$ in logarithmic scale. Its mean value is $\mu_{\log _{10}\left(E_{L T}\right)}=51.40$, and its standard deviation is $\sigma_{\log _{10}\left(E_{L T}\right)}=0.47$.

The LXRE power-law spread, given approximately by $2 \sigma_{\log _{10}\left(E_{L T}\right)}=0.94$, is larger than the one of the previous work of Pisani et al. (2013), which results as $2 \sigma_{\log _{10}\left(E_{L T}\right)}=0.56$. This is certainly due to the important growth of the number of BdHNe composing the ES (161) in respect to the ones of the GS (6).

Moreover, there is no evidence for a correlation between the LXRE power-law behavior and the isotropic energy radiated by the source during the GRB prompt radiation (for details, see Pisani et al. 2016). 


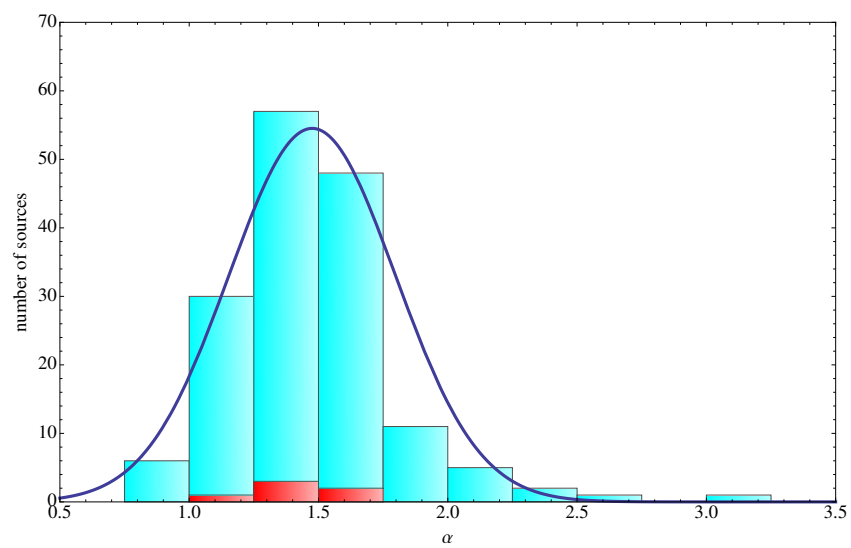

(a)

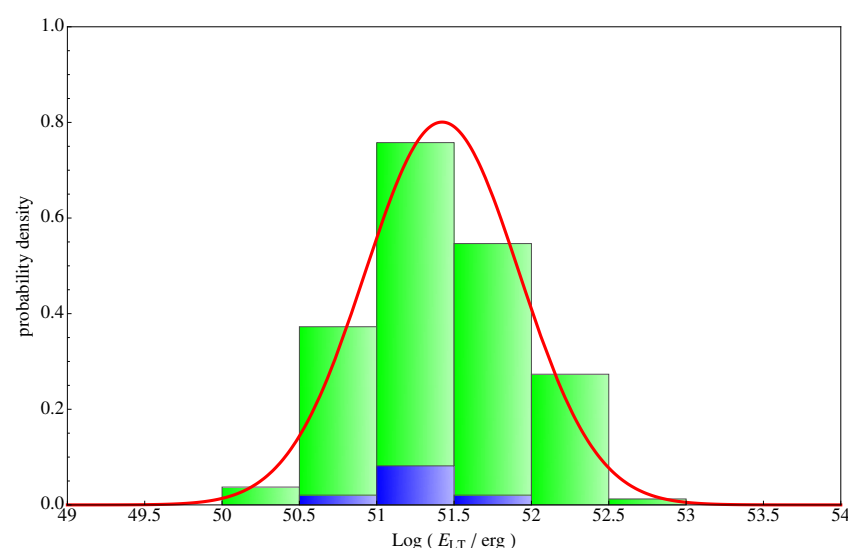

(b)

Figure 5. Panel (a): distribution of the LXRE power law indexes $\alpha$ within the ES (cyan) compared to the one of the GS (red). Such a distribution follows a Gaussian behavior (blue line) with a mean value of $\mu_{\alpha}=1.48$ and a standard deviation of $\sigma_{\alpha}=0.32$ (from Pisani et al. 2016). Panel (b): probability distribution of the LXRE integrated energies within the time interval $10^{4}-10^{6} \mathrm{~s}$ in the rest-frame after the initial GRB trigger for all the sources of the ES (in green) compared with the GS (in blue). The solid red line represents the Gaussian function which best fits the ES data in logarithmic scale. Its mean value is $\mu_{\log _{10}}\left(E_{L T}\right)=51.40$, while its standard deviation is $\sigma_{\log _{10}\left(E_{L T}\right)}=0.47$ (from Pisani et al. 2016).

\section{COLLIMATION}

In Pisani et al. (2016), we also proposed to reduce the spread of the LXRE power laws within the ES by introducing a collimation effect in the emission mechanism. In fact, if such a process is not isotropic, our estimates for the LXRE luminosities are actually overestimations of the intrinsic ones. By introducing a collimation effect, namely assuming that the LXREs are not radiated isotropically but inside a double-cone region having halfopening angle $\theta$, we can compute the intrinsic LXRE luminosity $L_{i n t r}\left(t_{r f}\right)$ from the isotropic $L_{i s o}\left(t_{r f}\right)$ :

$$
L_{i n t r}\left(t_{r f}\right)=L_{i s o}\left(t_{r f}\right)(1-\cos \theta) \text {. }
$$

From Eq. 7, an angle $\theta$ can be computed for each source of the ES if an intrinsec universal LXRE light curve $L_{\text {intr }}\left(t_{r f}\right)$ is given. By assuming GRB 050525A, which has the lowest luminosity within the ES, as our sole "isotropic" LXRE source, we obtain the probability distribution of the half-opening angle $\theta$ within the ES showed in Fig. 6a. The blue solid line represents a logarithmic normal distribution, which best fits the data. This distribution has a mode of $M o_{\theta}=17.62^{\circ}$, a mean of $\mu_{\theta}=30.05^{\circ}$, a median of $M e_{\theta}=25.15^{\circ}$, and a standard deviation of $\sigma_{\theta}=19.65^{\circ}$. In addition, it is possible to verify that, by adjusting the $L_{i s o}\left(t_{r f}\right)$ light curve of each ES source for its corresponding $\theta$, an overlap of the LXRE luminosity light curves as good as the one seen in the GS by Pisani et al. (2013) shown in Fig. 1 is obtained. Since the LXRE follows a power-law behavior, we can evaluate the tightness of the LXREs overlap estimating the correlation coefficient $\rho$ between all the luminosity light-curve data points of the ES sources in log-log scale. Considering the data points of the LXRE power laws within the $10^{4}-10^{6} \mathrm{~s}$ time interval (the time interval where we defined $E_{L T}$ ), we obtain $\rho=-0.94$ for the GS, $\rho=-0.84$ for the ES before the collimation effect correction, and $\rho=-0.97$ after the collimation correction. Therefore, assuming the collimation not only let the spread of the LXREs within the ES decrease, but makes the LXREs overlap even tighter than the one previously observed in the GS. This leads to the possible establishment of a new distance indicator, eventually useful to test the standard cosmological $\Lambda$ CDM model.

\section{THE CURRENT BDHNE CATALOG}

Thanks to the tremendous amount of work from ICRANet scientists in the past years (Ruffini et al. 2001a,b,c, 2007; Rueda \& Ruffini 2012; Izzo et al. 2012; Fryer et al. 2014; Ruffini et al. 2015, 2016), today we know that all the observed GRBs having long duration and isotropic energy $E_{\text {iso }}$ larger than $10^{52} \mathrm{erg}$ are the observational result of a BdHN phenomenon. Therefore, these two signatures are necessary and sufficient to identify a BdHN source. This holds also in the case it was not possible to observe the other typical features of the BdHNe following the GRB explosion, like: the FPA structure in the X-rays; the LXRE in the X-rays; and the associated $\mathrm{Ib} / \mathrm{c} \mathrm{SN}$ in the optical rays. In our recent work (Ruffini et al. 2017), in order to focus our analysis on the early X-Ray Flares in the FPA phase, we collected all the BdHNe ever observed till the end of 2016, collecting all the GRBs which satisfies the following criteria:

- measured redshift $z$;

- GRB rest-frame duration larger than $2 \mathrm{~s}$;

- isotropic energy $E_{i s o}$ larger than $10^{52} \mathrm{erg}$.

The updated list presented in Table 9 of (Ruffini et al. 2017) is composed by $345 \mathrm{BdHNe}$, and represents the current ICRANet catalog of BdHNe. The ES, updated to the end of 2016, counts 182 BdHNe having Swift/XRT data up to at least $10^{4} \mathrm{~s}$ in the rest-frame after the initial GRB explosion. It composes $\sim 53 \%$ of the total BdHNe catalog. Since the Swift satellite is operating since 2005, we have an average of $\sim 15 \mathrm{BdHNe}$ per year having good LXRE observations. Consequently, this representes the expected rate of $\mathrm{BdHNe}$ which, in the near future, will be useful to test the standard cosmological $\Lambda$ CDM model at redshifts up to $z \sim 8$.

This work made use of data supplied by the UK Swift Science Data Center at the University of Leices- 


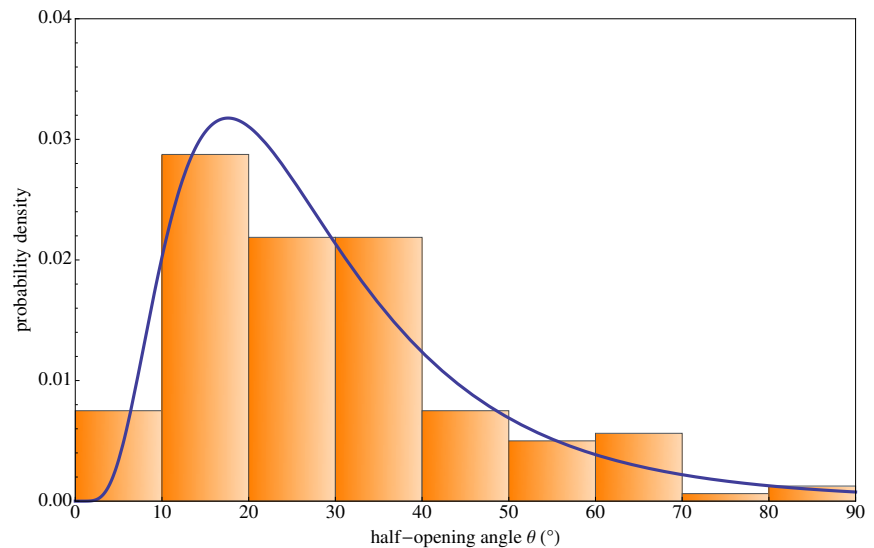

(a)

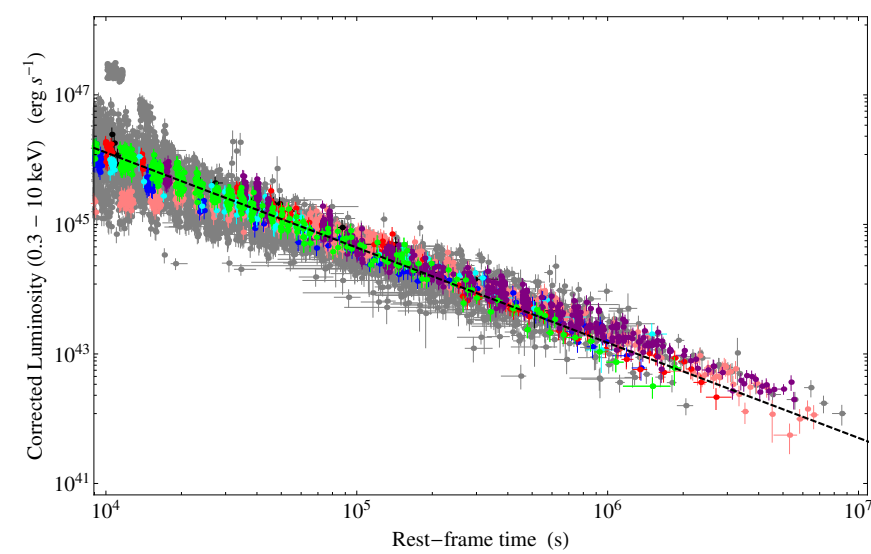

(b)

Figure 6. Left panel (a): probability distribution of the half-opening angle $\theta$ within the ES. The blue solid line represents a logarithmic normal distribution, which best fits the data. This distribution has a mode of $M o_{\theta}=17.62^{\circ}$, a mean of $\mu_{\theta}=30.05^{\circ}$, a median of $M e_{\theta}=25.15^{\circ}$, and a standard deviation of $\sigma_{\theta}=19.65^{\circ}$ (from Pisani et al. 2016). Right panel (b): corrected LXRE luminosity light curves of all 161 sources of the ES (gray) compared to the ones of the GS: GRB 060729 (pink), GRB 061007 (black), GRB 080913B (blue), GRB 090618 (green), GRB 091127 (red), and GRB 111228 (cyan), plus GRB 130427A (purple; Pisani et al. 2013; Ruffini et al. 2015). The black dotted line represents the universal LXRE power law, namely the linear fit of the late emission of GRB 050525A (from Pisani et al. 2016).

ter. J. A. R. acknowledges the support by the International Cooperation Program CAPES-ICRANet financed by CAPES-Brazilian Federal Agency for Support and Evaluation of Graduate Education within the Ministry of Education of Brazil. M. M. acknowledges the partial support of the project No. 3101/GF4 IPC-11/2015, and the target program of the Ministry of Education and Science of the Republic of Kazakhstan.

\section{REFERENCES}

Bloom, J. S., Frail, D. A., \& Sari, R. 2001, AJ, 121, 2879

Boella, G., Butler, R. C., Perola, G. C., et al. 1997, A\&AS, 122, 299

Costa, E., Frontera, F., Heise, J., et al. 1997a, Nature, 387, 783

Costa, E., Feroci, M., Piro, L., et al. 1997b, IAU Circ., 6576

de Pasquale, M., Piro, L., Gendre, B., et al. 2006, A\&A, 455, 813

de Ugarte Postigo, A., Xu, D., Leloudas, G., et al. 2013, GCN Circ., 14646, 1

Dezalay, J.-P., Barat, C., Talon, R., et al. 1992, in American Institute of Physics Conference Series, Vol. 265, American Institute of Physics Conference Series, ed. W. S. Paciesas \& G. J. Fishman, 304-309

Evans, P. A., Beardmore, A. P., Page, K. L., et al. 2007, A\&A, 469, 379

Evans, P. A., Willingale, R., Osborne, J. P., et al. 2010, A\&A, 519, A102

Frontera, F., Costa, E., Piro, L., et al. 1998, ApJ, 493, L67

Frontera, F., Amati, L., Costa, E., et al. 2000, ApJSS, 127, 59

Fryer, C. L., Rueda, J. A., \& Ruffini, R. 2014, ApJ, 793, L36

Gehrels, N., Chincarini, G., Giommi, P., et al. 2004, ApJ, 611, 1005

Izzo, L., Rueda, J. A., \& Ruffini, R. 2012, A\&A, 548, L5

Klebesadel, R. W. 1992, in Gamma-Ray Bursts - Observations, Analyses and Theories, ed. C. Ho, R. I. Epstein, \& E. E.

Fenimore (Cambridge University Press), 161-168
Kouveliotou, C., Meegan, C. A., Fishman, G. J., et al. 1993, ApJ, 413, L101

Levan, A. J., Fruchter, A. S., Graham, J., et al. 2013, GCN Circ., 14686, 1

Mazets, E. P., Golenetskii, S. V., Ilinskii, V. N., et al. 1981, Ap\&SS, 80, 3

Penacchioni, A. V., Ruffini, R., Bianco, C. L., et al. 2013, A\&A, 551, A133

Penacchioni, A. V., Ruffini, R., Izzo, L., et al. 2012, A\&A, 538, A58

Pisani, G. B., Izzo, L., Ruffini, R., et al. 2013, A\&A, 552, L5

Pisani, G. B., Ruffini, R., Aimuratov, Y., et al. 2016, ApJ, 833, 159

Rueda, J. A., \& Ruffini, R. 2012, ApJ, 758, L7

Ruffini, R., Bianco, C. L., Chardonnet, P., Fraschetti, F., \& Xue, S.-S. 2001a, ApJ, 555, L107

-. 2001b, ApJ, 555, L113

-. 2001c, ApJ, 555, L117

Ruffini, R., Bernardini, M. G., Bianco, C. L., et al. 2007, ESA SP 622,561

Ruffini, R., Bianco, C. L., Enderli, M., et al. 2013a, GCN Circ., 14526, 1

- 2013b, GRB Coordinates Network, 14888

—. 2013c, GRB Coordinates Network, 15576

— 2014a, GRB Coordinates Network, 15707

Ruffini, R., Izzo, L., Muccino, M., et al. 2014b, A\&A, 569, A39

Ruffini, R., Muccino, M., Bianco, C. L., et al. 2014c, A\&A, 565 L10

Ruffini, R., Wang, Y., Enderli, M., et al. 2015, ApJ, 798, 10

Ruffini, R., Rueda, J. A., Muccino, M., et al. 2016, ApJ, 832, 136

Ruffini, R., Wang, Y., Aimuratov, Y., et al. 2017, ArXiv e-prints

Salvaterra, R., Della Valle, M., Campana, S., et al. 2009, Nature, 461,1258

Tanvir, N. R., Fox, D. B., Levan, A. J., et al. 2009, Nature, 461, 1254

Tavani, M. 1998, ApJ, 497, L21 